\title{
At the crossroads of polarity, proliferation and apoptosis: The use of Drosophila to unravel the multifaceted role of endocytosis in tumor suppression
}

\author{
Thomas Vaccari, ${ }^{*}$, David Bilder* \\ Department of Molecular and Cell Biology, University of California, Berkeley, CA, USA
}

\section{A R T I C L E I N F O}

Article history:

Received 5 May 2009

Accepted 25 May 2009

Available online 6 June 2009

Keywords:

Endocytosis

Tumor suppression genes

Drosophila

Mitogenic signaling

Polarity

Apoptosis

Notch

EGFR

JNK

ESCRT

\begin{abstract}
A B S T R A C T
Endocytosis is an important regulator of cell-cell signaling and endocytic trafficking has been increasingly implicated in control of tumor suppression. Recent insights from Drosophila indicate that impairment of multiple trafficking steps which lead to receptor degradation can cause tumor formation in epithelial organs. These tumors are characterized by sustained activation of a number of mitogenic signaling pathways, and by subversion of epithelial polarity and the apoptotic response. Cooperation between such alterations, as well as tumor-host interactions, is also observed. The recapitulation of several hallmarks of human cancers in fly tumors provides a framework to understand the role of defective endocytosis in cancer.
\end{abstract}

(c) 2009 Federation of European Biochemical Societies. Published by Elsevier B.V. All rights reserved.

\section{Introduction}

Cancer progression involves the stepwise acquisition of a number of neomorphic traits by tumor cells. These traits include self-sufficiency in growth signaling, evasion of apoptosis, insensitivity to antigrowth signals, tissue invasion and metastasis, and the ability to alter tumor environment (Hanahan and Weinberg, 2000). These hallmarks of cancer require cells to subvert a number of physiological processes that together contribute to determine the overall growth and organization of tissues. Processes generally subverted by cancer cells include cell proliferation, cell polarization and programmed cell death. These processes are genetically controlled by a number of tightly regulated, widely conserved signaling pathways that communicate the state of cells within a tissue. For this reason, genes that act as negative regulators of intercellular signaling pathways can behave as tumor suppressors.

\footnotetext{
* Corresponding authors. Tel.: +39 0257430 3823; fax: +39 02574303231 (T. Vaccari), Tel.: +1 510642 8605; fax: +1 5106437440 (D. Bilder). E-mail addresses: thomas.vaccari@ifom-ieo-campus.it (T. Vaccari), bilder@berkeley.edu (D. Bilder).

${ }^{1}$ Present address: IFOM, Istituto FIRC di Oncologia Molecolare, Milano, Italy. 1574-7891/\$ - see front matter ( 2009 Federation of European Biochemical Societies. Published by Elsevier B.V. All rights reserved. doi:10.1016/j.molonc.2009.05.005
} 
Since cancer cells promote their own growth and dissemination by altering physiological events that normally occur in the tissue of origin, the question of how polarity, growth and apoptosis are controlled and can be subverted is one of central importance to modern cancer biology. Unfortunately, answering this question in mammalian cells is complicated by the frequent functional redundancy of mammalian genes as well as the extensive crosstalk between different pathways. Drosophila has more simple genetic circuitry and has been extensively used to study polarity, growth, and apoptosis, in particular due to the ability to perform genetic screens to identify new molecules involved in a given pathway (StJohnston, 2002). In addition, a good proportion of cancer and disease genes in humans are conserved in Drosophila and, in many case, a single fly gene serves the function of multiple related mammalian family members (Fortini et al., 2000; Bernards and Hariharan, 2001). As extensively reviewed elsewhere, the exploitation of these features is turning the fly into an outstanding genetic system to study many biological processes that are relevant to tumorigenesis (Bilder, 2004; Hariharan and Bilder, 2006; Brumby and Richardson, 2005).

Endocytosis, the process by which cells internalize different kinds of molecules, has been increasingly implicated in controlling tumor suppression (Mosesson et al., 2008). In this review, we will focus on emerging insights from Drosophila suggesting that growth, polarity and apoptosis in epithelial organs is strongly altered in tumors that arise when endocytic regulators are impaired. These tumors display several hallmarks of cancer and, as discussed at the end of the review, might represent useful cancer models.

\section{The dawn of tumor suppression studies in Drosophila}

The origins of tumor biology in Drosophila can be traced back almost half a century, when Schneiderman, Gateff, and Bryant identified mutations in a group of genes that they called 'tumor suppressor genes' (TSGs). Animals homozygous mutant for TSGs contained tumor-like tissue growths in larval and/or adult organs. Larval tumors occurred in several tissues including in the brain, the hematopoietic system, and the developing epithelial organs called imaginal discs. The tumorous tissue displayed continuous proliferation, and, in some cases, altered cell morphology. In addition, some of the tumors were invasive and transplantable, and the transplanted tumors were lethal to the recipient as they were to the original host. These and other characteristics indicated that the fly tumors displayed hallmarks of cancer, and satisfied several criteria for tumors used by vertebrate cancer biologists (Gateff and Schneiderman, 1967; Bryant and Schubiger, 1971; Gateff, 1978).

Since their original isolation, characterization of the different fly TSG mutations has revealed a wealth of tumor tissue phenotypes. For instance, tumors arising from epithelial imaginal disc tissue have been sorted in two classes. The first class includes 'hyperplastic' tumors, which display increased proliferation but otherwise normal differentiation and organ architecture. The second class includes 'neoplastic' tumors, which displayed not only overproliferation but also altered epithelial architecture and loss of terminal differentiation. Despite their epithelial origin, tumor cells lack cell-cell junctions and have a disorganized appearance, bringing to mind the epithelial to mesenchymal transition (EMT) observed during malignant cell transformation in human cancer. The original neoplastic TSG mutations disrupt scribble, discs large, lethal (2) giant larvae, which encode three proteins that function as scaffolds at cellcell junctions and which show altered expression in certain human cancers (Bilder et al., 2000; Humbert et al., 2003).

The limited number of mutations initially identified that lead to tumorous growths in homozygous flies, as compared to the variety of known mammalian TSGs, could reflect the lethality of these mutations during early development. However, genetic mosaic technology was subsequently developed, which allows the generation of homozygous mutant tissue within an otherwise heterozygous fly by mitotic recombination. This technological advancement was instrumental in developing genetic screens that have now identified a much larger group of TSGs, including members of both the hyperplastic and neoplastic classes. These TSGs have dramatically expanded our understanding of the mechanisms that control cell proliferation and restrain organ size.

\section{Endocytic genes define a novel type of Drosophila tumor suppressors}

Mosaic screens aiming at identifying new neoplastic TSGs were designed to recover mutations that were lethal to the larva when homozygous mutant cells were generated within only a small amount of epithelial tissue. This design was meant to both circumvent the limitations of previous screens (see above) and to mimic the clonal origin of cancer in vertebrate epithelia. Mosaic screening led to a four-fold increase in the number of known neoplastic TSGs (Lu and Bilder, 2005; Vaccari and Bilder, 2005; Menut et al., 2007; Morrison et al., 2008). Surprisingly, despite the unbiased nature of the approach, genetic mapping of the majority of the newly isolated TSG mutations revealed lesions in genes coding for a single class of proteins: regulators of the endocytic pathway.

Endocytosis is the main process by which cells maintain transmembrane protein homeostasis. Endocytic trafficking involves internalization of portions of the plasma membrane to form carrier vesicles. Multiple routes of internalization exist in cells, which differ in their requirements for core cellular machinery such as the hexameric Clathrin membrane coat and the small GTPase Dynamin involved in vesicle scission (for review, Doherty and McMahon, 2009). Most of these routes lead to fusion of vesicles to the so-called 'early endosome', in a process requiring specific syntaxins and regulated by the small GTPase Rab5. The early endosome serves as a station for both recycling certain cargoes back to the plasma membrane, and for sending others for degradation in the lysosome (Spang, 2009). Sorting for degradation involves capturing of ubiquitylated cargoes on the limiting membrane of endosomes by the Hrs/STAM complex and subsequent sequestration to internal luminal vesicles by components of three Endosomal Sorting Complex Required for Transport (ESCRTI, -II, -III) complexes. This leads to the formation of multivesicular bodies (MVB), which fuse with lysosomes in which acidification-dependent degradation of internal luminal vesicles and their embedded cargoes occurs (Saksena et al., 2007). 

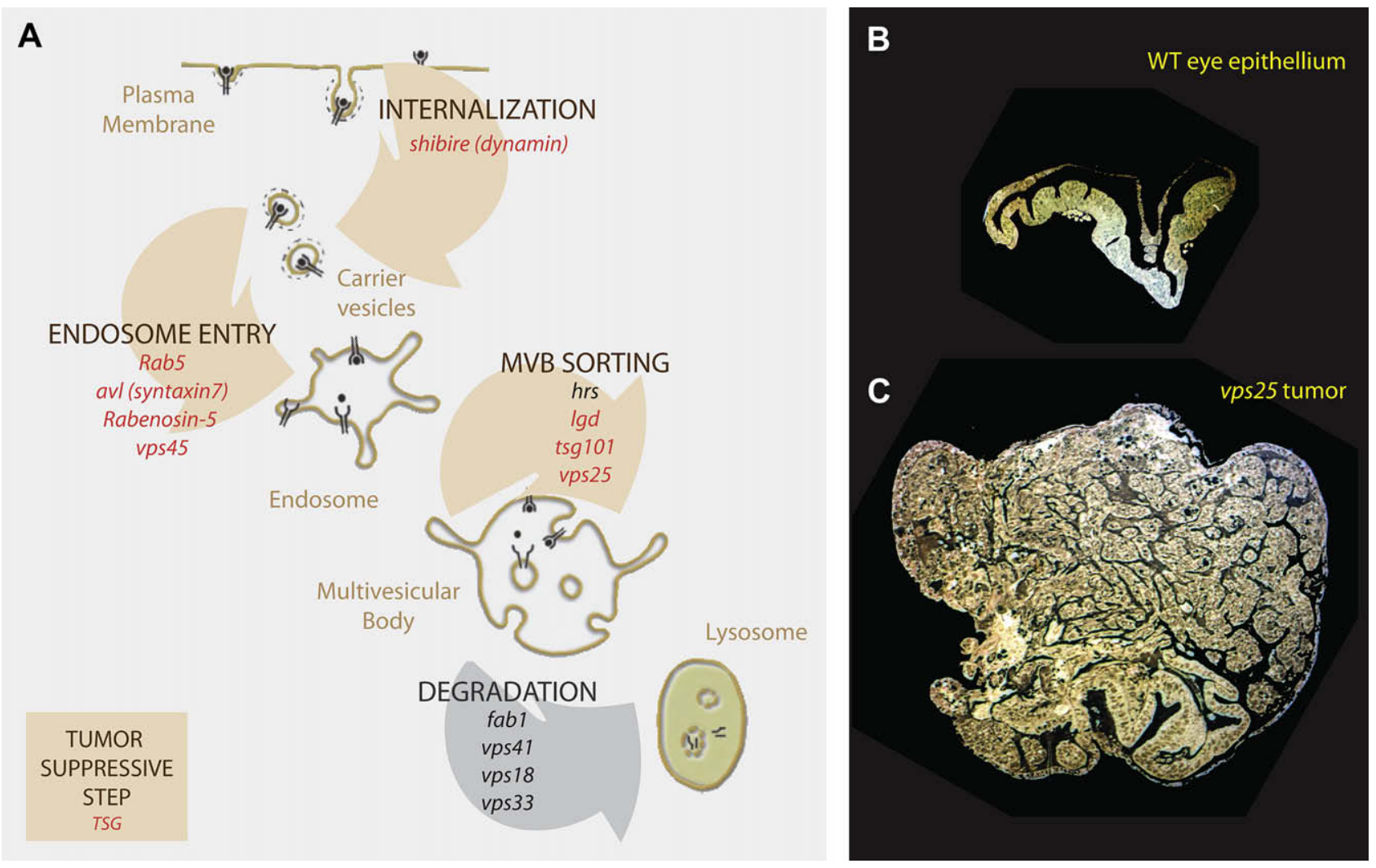

Figure 1 - Tumorigenic steps of endocytosis. (A) Drosophila tumors arise when multiple steps of endocytic trafficking are blocked. Schematic view of the compartments composing the endocytic system of Drosophila epithelial cells and of the endocytic trafficking steps that control tumorigenesis. The genes that act as tumor suppressors at the step of internalization of cargoes, entry of cargoes into endosomes and ubiquitylation-dependent sorting of cargoes into MVBs are listed in red. Genes that do not act as tumor suppressors are listed in black. (B) Loss of epithelial architecture and growth control in endocytic tumors. Cross-section of the epithelial primordia of the eye in WT Drosophila larvae (top) and of tumorous eye primordia arising from lack of $v$ ps25, an ESCRT component crucial of MVB sorting of ubiquitylated cargoes (bottom). Note the monolayered organization of the WT epithelial tissue, compared to the multilayered and overgrown organization of the tumor (same magnification).

The endocytic genes known to behave as TSGs in Drosophila encode regulators of two of these steps: cargo entry into the early endosome and cargo sorting within the MVBs (Figure 1). The former genes encode the fly homologs of Syntaxin7/12 [called Avalanche (Avl) in Drosophila], the GTPase Rab5, the Syntaxin-binding protein Vps45 and the Rab5 effector Rabenosyn-5. The latter encode Tsg101 and Vps28, two components of the ESCRT-I complex, Vps25 and Vps22, two components of the ESCRT-II complex, and Vps20, Vps32 and Vps2, three components of the ESCRT-III complex (Lu and Bilder, 2005; Moberg et al., 2005; Thompson et al., 2005; Vaccari and Bilder, 2005; Herz et al., 2006; Morrison et al., 2008; Vaccari et al., in press). Screens have not been saturating and approximately half of the Drosophila genome has not been screened yet, suggesting that the TSGs above constitute only a fraction of the endocytic genes that control growth in Drosophila.

Null mutants in all of the above endocytic regulators lead to formation of neoplastic tumors characterized by similar loss of epithelial polarity and of organ growth. At the cell biological level, electron microscopy and immunohistochemistry also reveal major defects in endocytic compartment morphology, ranging from accumulation of small vesicles below the plasma membrane of Aul, Rab5, ups45, and rabenosyn-5 mutant cells to enlarged endosomes and impaired MVB formation in ESCRT mutant cells
(Morrison et al., 2008; Vaccari et al., in press). Endocytic tracer uptake and cargo trafficking assays were conducted in mutants to determine whether endocytic trafficking was altered. Such experiments reveal that endocytic trafficking is blocked at the step of endocytosis at which the mutated gene is expected to function (Lu and Bilder, 2005; Vaccari and Bilder, 2005; Morrison et al., 2008; Vaccari et al., 2008). Together with the fact that all the mutations are predicted to express non-functional protein truncations, such trafficking defects suggest that the neoplastic tumors arise from complete lack of endocytic gene function.

The discovery that endocytic trafficking steps are tumor suppressive by forward genetics was rapidly followed by reports that a classical Drosophila TSG with then unknown function, called lethal giant discs (lgd), also plays a role in endocytic trafficking (Childress et al., 2006; Gallagher and Knoblich, 2006; Jaekel and Klein, 2006). A role in endocytic trafficking also emerged for the Drosophila TSGs merlin and expanded, proteins related to that encoded by the human TSG NF2 (Maitra et al., 2006). In addition, shibire, the Drosophila homolog of dynamin, a well-studied endocytic component, was found to act as a TSG (Vaccari et al., 2008). This evidence reinforced the idea that impairment of endocytosis can be a direct cause of tumor induction in Drosophila. Interestingly, analysis of other endocytic mutants suggests that impairment of certain endocytic steps 
do not lead to tumor formation. This is the case of Drosophila mutant tissue for the late-acting endocytic mutants such as the late endosomal PtdIns(3)P 5-kinase/PIKfyve Fab-1 and for lysosomal HOPS complex genes ups18, ups33 and ups41 (Shestopal et al., 1997; Warner et al., 1998; Rusten et al., 2006).

Together, the mosaic screens and subsequent research summarized above establish the existence of a novel class of Drosophila tumor suppressor genes, which we will refer here as to 'endocytic TSGs'. The evidence that endocytic steps that precede cargo sequestration into MVBs are tumor suppressive in Drosophila suggests that these genes act as tumors suppressors by preventing inappropriate trafficking and accumulation of cargoes important for polarity and growth in epithelial tissues.

\section{Jack of all trades: the endosomal system is as a multivalent infrastructure for diverse cellular processes}

In order to understand how mistrafficking of cargoes might alter tissue growth and polarity, it is useful to consider how endocytosis controls these processes in the first place. Endocytosis plays a well-established and conserved role in controlling signal transduction, as reviewed elsewhere in this issue. Thus, here we will only touch on the current understanding of how endosomes are thought to control signaling, and briefly review evidence in Drosophila useful to later discussions of the phenotypes of endocytic TSG mutant cells.

A classical paradigm posits that endocytosis acts as a negative regulator of signaling because a prominent fate of receptors that are trafficked away from the plasma membrane is MVB sorting and lysosomal degradation. However, a growing body of studies in different organisms indicates that endocytic trafficking can also sustain or even activate signaling (Miaczynska et al., 2004). The emerging role of the endosome in activating signaling is illustrated in Drosophila by studies on Notch, TGF-beta [Decapentaplegic (Dpp) in Drosophila], Wnt [wingless (wg) in Drosophila] and Jak/Stat signaling. Indeed, following ligand engagement, entry of the Notch receptor in endosomes appears necessary for signaling activation (reviewed in (Fortini and Bilder, in press; Kopan and Ilagan, 2009; Tien et al., 2009)). Interestingly, very recent data suggest that in specific developmental contexts, Notch is activated in a specific subpopulation of endosomes containing the endocytic protein SARA, which have been previously characterized as a major site of Dpp signaling (Bökel et al., 2006; Coumailleau et al., 2009). As for Wg, while several studies indicated that signaling is attenuated by lysosomal degradation (Dubois et al., 2001; Piddini et al., 2005; Marois et al., 2006; Rives et al., 2006), one report suggests that Wg signaling is indeed activated in endosomes (Seto and Bellen, 2006); Finally, Jak/ STAT signaling activation is dependent on endocytic trafficking of Domeless, the IL- 6 related receptor for Jak/STAT signaling (Devergne et al., 2007). This evidence implies that the signaling profile of endocytic TSG mutant cells is likely to depend on the specific step of endocytosis affected and the activation status of each signaling pathway at such step.

The widespread role of endocytosis in control of signaling suggests the possibility that loss of tumor suppression in endocytic TSG mutant cells might arise from altered growth and polarity signaling. In contrast to the variety of gene product interference strategies and cellular contexts in which growth and polarity alterations have been studied in mammals, a large part of these studies in Drosophila have been conducted in epithelial imaginal discs utilizing null mutant alleles. This has provided a fairly uniform context in which to accurately compare evidence to unearth some of the circuitry of endocytic tumors, as discussed in the next part of this review.

\section{Clogging the mitogen sink: multiple receptors fail to be degraded and cannot stop signaling in endocytic TSG mutant tissue}

One of the prominent features of endocytic tumors in Drosophila is their unrestrained proliferation. For instance, aul tumors can be composed of almost three times as many cells as the epithelial tissue they originate from by the time they are lethal to the host (Lu and Bilder, 2005); ESCRT mutant tumors appear even larger. Such overproliferation implies that the mutant cells have acquired self-sufficiency in growth signaling. How could such a trait be acquired in tumors arising from impaired endocytic function? Not surprisingly, given the role of endocytosis in controlling multiple signaling pathways, self-sufficiency is likely to be acquired by ectopic activation of more than one mitogenic signal. These include, in the order discussed below, pathways involving EGFR, Notch, and dpp. A contribution of Jak/STAT and Wg signaling is also likely but merits further testing (Figure 2).

RTK signaling is widely used by multiple cell types to control proliferation in different organisms. In Drosophila as in mammals, signaling induced by activation of EGFR is downregulated by ubiquitylation and MVB-mediated degradation of EGFR (Pai et al., 2000; Jékely et al., 2005). Consistent with an impairment of EGFR degradation, clones of ESCRT mutant cells accumulate EGFR in endosomes. This accumulation correlates with sustained EGFR signaling, as also assessed by genetic interactions with aul and ESCRT mutants (Vaccari et al., in press). Despite the fact that EGFR signaling activation has not been systematically tested in all endocytic TSG mutants, this evidence suggests that sustained EGFR signaling activation might contribute to some of the self-sufficiency in growth signaling observed in Drosophila endocytic tumors.

A second way by which certain Drosophila endocytic tumors could reach self-sufficiency in growth signaling is by ectopically activating Notch signaling. In contrast to EGFR signaling, the extent of Notch signaling activation has been systematically analyzed in Drosophila endocytic TSG mutant tissue. In imaginal discs, mutations of endocytic TSGs that block MVB sorting of Notch, such as lgd and ESCRT $\backslash$ components, as well as expression of a constitutively active Rab5, lead to endosomal Notch accumulation and to high expression of reporters of Notch signaling activation (Moberg et al., 2005; Thompson et al., 2005; Vaccari and Bilder, 2005; Childress et al., 2006; Herz et al., 2006; Jaekel and Klein, 2006; Vaccari et al., 2008). Considering that expression of a constitutively active Notch in eye imaginal discs per se is mitogenic and results in overproliferation (Chao et al., 2004), ectopic Notch activation in endocytic TSGs that block MVB sorting is likely to contribute to growth self-sufficiency, although this has not yet been directly tested. In sharp contrast, mutations of endocytic TSGs such as shibire, aul, Rab5, ups45 and rabenosyn-5, or overexpression of a dominant negative 


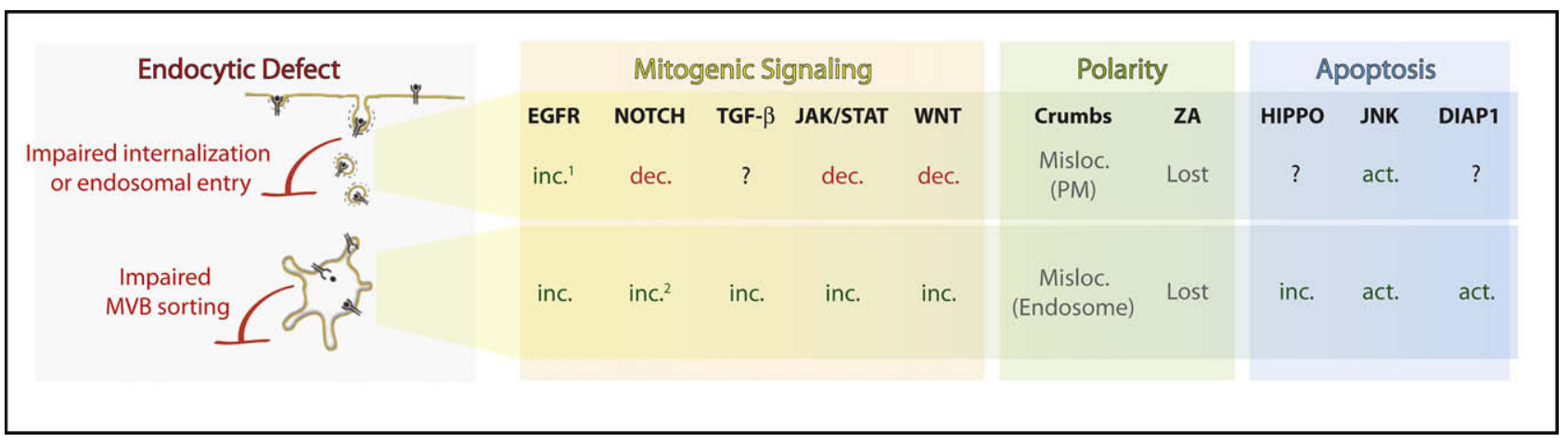

Figure 2 - The mitogenic, polarity and apoptotic profile of endocytic TSG tumors. Schematic representing the alterations observed in tumors displaying impaired cargo internalization or entry into the early endosome (top row) or impaired MVB sorting (bottom row). The mitogenic (yellow), polarity (green) and apoptotic (light blue) pathways found altered are listed. Abbreviations: Inc. $=$ increased signaling, Dec. $=$ decreased signaling Act. = activated signaling, ZA Misloc. $=$ Mislocalized, $P M=$ plasma membrane. $1=$ stronger increase in mutants affecting cargo internalization or entry into the early endosome, rather MVB sorting. $2=$ ligand-independent activation. See text for details.

form of Rab5 lead to failure to activate the Notch receptor (Lu and Bilder, 2005; Morrison et al., 2008; Vaccari et al., 2008), indicating that signaling is not active in endocytic TSGs that control entry of Notch into the early endosome. This evidence suggests that mitogenic Notch signaling is not required for overproliferation in this subset of endocytic TSG mutants.

Notch signaling activation in endosomes of cells that lack lgd and ESCRTs is ligand-independent (Thompson et al., 2005; Childress et al., 2006; Jaekel and Klein, 2006; Vaccari et al., 2008). The mechanism of this unusual mode of Notch activation in lgd and ESCRTs mutant tissue is presently unclear. However, ligand-independent Notch activation happens physiologically in a subset of normal Notch signaling events regulated by the E3 ubiquitin ligase Deltex (Wilkin et al., 2004; Fuwa et al., 2006). Deltex directs Notch to the degradative late endosomal/ lysosomal compartment and acts with the B-arrestin Kurtz to degrade it (Hori et al., 2004; Mukherjee et al., 2005). Recent data indicate that HOPS and AP-3 complexes in certain contexts act in opposition to MVB sorting and shunt Notch to the limiting membrane of endosomes allowing activation of unliganded Notch (Wilkin et al., 2008). Considering that to maintain a steady-state levels of Notch at the plasma membrane, a portion of unliganded and inactive Notch is continuously produced and targeted to degradation via endocytic trafficking, (Sakata et al., 2004), and that endosomal pH is optimal for activity of $\gamma$-secretase, the enzyme that cleaves and release active Notch (Pasternak et al., 2003), it will be interesting to understand whether impaired sorting of Notch in lgd and ESCRT mutant cells might expose the accumulated unliganded Notch to an acidifying and cleavage-prone environment, thus favoring ectopic and ligand-independent Notch cleavage and activation. This mechanism might support some of the self-sufficiency in growth signaling observed in lgd and ESCRTs tumors.

Other signaling pathways, though less studied, could also contribute to self-sufficiency in growth signaling. In one example, cells lacking Vps25 display endosomal accumulation of Thickveins, one of the receptor for Dpp signaling, which is known to be active in endosomes (Thompson et al., 2005; Bökel et al., 2006). In addition, a reporter for Dpp signaling activation is highly expressed in clones of ups 25 mutant cells and some of the outgrowth of surviving ups25 mosaic animal mimicks those obtained by ectopic dpp activation (Thompson et al., 2005). In a second example, evidence suggests that overproliferation in certain Drosophila endocytic tumors might be mediated by Jak/STAT signaling. Indeed, ectopic Notch signaling activation in ESCRT mutant cells causes inappropriate expression of Unpaired (Upd), a potent mitogen during normal growth of eye imaginal discs (Tsai and Sun, 2004; Moberg et al., 2005; Vaccari and Bilder, 2005; Herz et al., 2006). Upd is the secreted ligand for Jak/STAT signaling, whose activation perdures in endosomes (Devergne et al., 2007). Since Upd accumulates in ESCRT mutant endosomes, it is possible that impaired degradation of Jak/STAT signaling components in ESCRT tumors might cause sustained autocrine activation of signaling. However, while paracrine Jak/STAT signaling activation is sufficient to sustain non-cell-autonomous growth (Moberg et al., 2005; Vaccari and Bilder, 2005; see cooperativity section), the contribution of autocrine Jak/STAT signaling to the growth of ESCRT tumors remains to be assessed.

Finally, while accumulation of $\mathrm{Wg}$ receptors has not been tested in ESCRT mutant cells, Wg signaling activation is reduced in cells in which Dynamin or Rab5 function is impaired, and enhanced in hrs mutant cells (Seto and Bellen, 2006), suggesting that ectopic Wg signaling might be present in ESCRT tumors. Overall, the analysis of mitogenic signaling in Drosophila endocytic tumors indicates that multiple pathways are altered. Further research will reveal the contribution of each pathway to the tumor phenotype.

\section{The ying and yang of epithelia: the} interdependence of endocytic TSG function and cell polarity

Epithelial polarity, like intercellular signaling, relies on cues received from the extracellular environment. Therefore, it might be expected that endocytosis of cell surface proteins could play a role in epithelial polarity as well. Mammalian cell culture experiments initially stressed the role of polarized sorting and delivery of TM proteins in PM polarity. Recently, 
however, genetic screens in flies have pointed to a central role for endocytosis as well in this process. Many mutations that confer strong blocks in the endocytic pathway, whether at the cell surface, entry into early endosomes, or sorting into MVBs, display a common defect in epithelial polarity. In these mutant cells, peripheral membrane proteins normally restricted to the apical membrane domain, such as aPKC, are mislocalized instead throughout the plasma membrane (Lu and Bilder, 2005; Vaccari and Bilder, 2005; Morrison et al., 2008). This phenotype suggests an 'apicalization' of the plasma membrane highly reminiscent of that seen in cells mutant for TSGs that act as scaffold at cell-cell junctions (Bilder et al., 2000). As mentioned above, these endocytic mutant cells also show cell-autonomous overproliferation to form neoplastic tumors, although there appear to be differences in proliferation kinetics and cell survival amongst the endocytic mutant cells, perhaps due to the additional signaling pathways differentially activated in them (see above and below).

This dramatic demonstration of the importance of endocytosis in controlling epithelial polarity suggests that cell surface levels of critical polarity regulators must be tightly controlled to allow proper maintenance of epithelial organization. An attractive candidate for such a regulator is Crumbs, which is a transmembrane protein that displays characteristics of an apical membrane determinant in Drosophila tissues. Increasing Crumbs levels by transgenic overexpresion apicalizes cells and drives neoplastic transformation (Lu and Bilder, 2005); a similar increase resulting from a failure in endocytosis could cause the same phenotype. The involvement of endocytic regulation of Crumbs or other TM cargo in neoplastic transformation has not yet been directly tested. Moreover, levels of Crumbs appear increased in ESCRT mutant cells, but most of the Crb is trapped in aberrant endosomes (Moberg et al., 2005; T.V. and D.B., unpublished) it is not clear how accumulation of this internal store of Crb could induce the PM polarity defects.

The striking phenotypic similarities between mutations in endocytic regulators and the junctional scaffold proteins suggest that there may be molecular links between these two classes of neoplastic TSGs. Could the junctional scaffold nTSGs control proliferation through effects of endocytosis? While the mechanistic activity of the junctional scaffold nTSGs in epithelial polarity remains unclear, it has been established that they act by inhibiting the Par complex proteins which control apical membrane identity. Interestingly, several recent reports have highlighted activities of the Par complex, along with the small GTPase Cdc42, in regulating endocytosis in general. In Caenorhabditis elegans and mammalian cells, these proteins promote either endocytic internalization or recycling of several different cargo (Balklava et al., 2007). A role for Par proteins in promoting surface endocytosis has also been described in Drosophila imaginal discs, where the mechanism seems to involve actin polymerization (Georgiou et al., 2008; Leibfried et al., 2008). However, in Drosophila embryos, Par proteins and Cdc42 seems to negatively regulate endocytosis from the cell surface (Harris and Tepass, 2008). The precise step of endocytosis, and the endocytic regulator, which the Par complex controls is therefore not yet clear. Overall, whether junctional scaffold nTSGs have according effects on endocytosis, and whether an endocytic defect underlies the common polarity and overgrowth phenotypes seen in the TSG mutant tissue, remains unclear.
While the reasons for which loss of epithelial polarity causes overproliferation in Drosophila remains unknown, one possibility is that disruption of polarity and cell-cell junctions creates a situation in which contact inhibition of proliferation is lost. Contact inhibition of proliferation is a phenomenon originally defined in mammalian cell culture, in which confluent cells that are untransformed will stop proliferating; however, transformed cells will continue to proliferate and pile up upon each other. The extent to which the mechanisms underlying disrupted contact inhibition in culture reflect the mechanisms disrupted in a human tumor is the subject of debate. Nevertheless, it is striking that a major regulator of contact inhibition is the Merlin protein, encoded by the human TSG NF2, which is mutated in some neurofibromatosis patients (Johnson et al., 2002; Curto et al., 2007). Merlin is a cytoskeleton-associated protein found at cell-cell junctions, and primary cultured cells mutant only for NF2 show a strong disruption of contact inhibition. The mechanisms underlying NF2-mediated tumor suppression are the subject of a recent review (McClatchey and Fehon, 2009), but in this context it is notable that one defect of NF2 mutant cells is increased endocytic traffic of EGFR. Upon cell-cell contact, Merlin seems to sequester EGFR in an endocytosis-resistant membrane domain, contributing to silencing of EGFR signaling. Conversely, NF2 mutant cells show increased EGFR endocytosis as well as increased signaling that promotes cell proliferation, even in confluent cultures (Curto et al., 2007). Interestingly, in fly tissues, where Merlin also participates in growth control, loss of Merlin and the related protein Expanded results in decreased endocytosis of EGFR and several other signaling receptors (Maitra et al., 2006). The link seen with Merlin between cell-cell contacts, endocytosis, and proliferation control provides further support that these links are not limited to the fly but also play a role in mammalian cancer biology.

\section{Dead or alive? The ambivalent regulation of apoptosis in endocytic TSGs mutant tissue}

Polarity alterations in epithelial cells are known to trigger apoptosis. Despite this, one of the hallmarks of cancer cells is that they are able to escape apoptotic elimination. Is apoptosis a feature altered in endocytic TSG tumors? Remarkably, endocytic TSG mutant cells display a differing apoptotic behavior that depends on tissue context in which the tumors arise. Consistent with their loss of epithelial architecture and of cell-cell junctions, it was found that, when clones of mutant cells are surrounded by WT cells, they are apoptotic and eventually eliminated from the epithelium (Lu and Bilder, 2005; Thompson et al., 2005; Vaccari and Bilder, 2005; Herz et al., 2006; Morrison et al., 2008). A recent study focusing on apoptosis of ups $25 \mathrm{mu}$ tant tissue concludes that there are at least two proapoptotic pathways activated in these cells: One that is mediated by the JNK signaling and another that is mediated by the Hid/Diap1/ Dronc/Ark (Herz et al., 2006). When both pathways are blocked, clones of ups 25 mutant cells become extremely overgrown, indicating that even though mitogenic pathways are activated in these cells (see above), their growth potential is also heavily hindered by activation of multiple apoptotic pathways.

Interestingly, activation of the Hippo pathway is increased in clones of ups 25 mutant cells. Significantly, inactivation of 
Hippo signaling completely blocks cell death in ups25 mutant clones, suggesting that the Hippo pathway control overall apoptotic response in these cells (Herz et al., 2006). Hippo signaling can induce cell death (reviewed by Edgar, 2006; Vidal and Cagan, 2006), but it is currently unknown how Hippo signaling is activated in ups25 mutant cells. By analogy to Notch, it has been proposed that a putative receptor which controls Hippo signaling may fail to be degraded in ups 25 mutant cells, leading to overactivation of Hippo signaling. The possibility of aberrant Hippo pathway activation in endocytic TSG mutant tissue is particularly intriguing because the Hippo pathway controls a wide range of cellular functions including, in addition to cell death, cell growth and polarity (Hamaratoglu et al., 2006; Willecke et al., 2006). Disruption of the Hippo pathway might therefore potentially link the apoptotic, proliferative and polarity aspects of the endocytic tumors (see below).

In sharp contrast with the behavior of clones of mutant cells that neighbor WT cells, when the entire imaginal disc is mutant, cells are not apoptotic. However, they still strongly activate JNK signaling, as judged by ectopic expression of MMP-1 (Menut et al., 2007; Morrison et al., 2008; Vaccari et al., in press), a JNK target (Adachi-Yamada et al., 1999). The mechanism of such opposite apoptotic behavior that depends on tumor context is unknown. However, it is interesting to note that JNK-mediated apoptosis is activated in response to signaling discontinuities, such as those likely to occur at the boundary between WT and endocytic mutant tissue (Adachi-Yamada and O'Connor, 2002). It is thus possible that endocytic mutant cells that are part of an entirely mutant imaginal discs or that lay at the center of large tumors might escape apoptotic elimination. In addition, it has been recently been suggested that JNK activation depends on endocytic trafficking of Eiger, the Drosophila homolog of tumor necrosis factor (TNF) (Igaki et al., 2009). These data suggest the possibility that apoptotic response of endocytic mutant cells might also be dictated by trafficking of specific apoptotic cargoes.

\section{Partners in crime: cooperative pathway interactions and heterotypic signaling in Drosophila endocytic TSG mutant tissue}

Given the variety of mitogenic, cell death and polarity pathways that are altered in endocytic TSG mutant cells (see above), is cooperation among these pathway contributing to the tumor phenotype? A recent line of investigation provides evidence supporting this scenario in tumors arising from lack of scaffolding TSG function. Indeed, these become invasive and metastatic upon the additional forced activation of either Ras, a key transducer of RTK signaling, or of Notch signaling (Brumby and Richardson, 2003; Pagliarini and $\mathrm{Xu}, 2003)$. Subsequent studies suggest that in such a genetic context, loss of cell polarity, combined with strong mitogenic signaling, trigger a switch in the role of JNK signaling from proapoptotic to progrowth/proinvasive (Igaki et al., 2006; Uhlirova and Bohmann, 2006). As specified above, most endocytic TSG tumors display loss of polarity similar to that of scaffolding TSGs. In addition, they activate JNK signaling and display ectopic EGFR signaling. Finally, endocytic TSGs that block MVB sorting, such ESCRT strongly activate Notch signaling and display metastatic behavior upon block of apoptosis (Thompson et al., 2005). This evidence predicts that cooperative interactions between mitogenic, cell death and polarity pathways might contribute to the endocytic TSG tumor phenotype. Such indirect evidence provides the basis for future investigation of whether and how endocytosis controls invasive and metastatic behavior, two of the deadliest hallmarks of cancer.

A final hallmark of vertebrate cancers that has received renewed attention is heterotypic signaling, which is the ability of cancer cells to modify the environment in which they arise and viceversa (reviewed in Bissell and Radisky, 2001). Interestingly, certain Drosophila endocytic tumors display two forms of heterotypic signaling. One form of heterotypic signaling in Drosophila involves paracrine secretion of Upd from ESCRT mutant cells. This process activates Jak/STAT signaling in neighboring WT cells. As a result, these cells overproliferate to form hyperplastic tissue surrounding the ESCRT tumors (Moberg et al., 2005; Vaccari and Bilder, 2005; Herz et al., 2006). A second form of heterotypic signaling involves enhancement of cell survival in tissue surrounding ESCRT tumors. Indeed, ESCRT mutant cells promote non-cell-autonomous Diap1 expression by an unknown mechanism. This signaling behavior is unrelated to the first pro-proliferative signaling, since the former does not depend on overactivation of Notch signaling (Herz et al., 2006). Overall, the ability to model cooperative and heterotypic tumor interactions in Drosophila promises to shed light on the rules governing tumor-host signaling.

\section{The emerging field of endocytic tumorigenesis in Drosophila}

Based on the first hints of the genetic circuitry of the endocytic tumors reviewed above, a speculative model to explain the role of endocytosis in tumor suppression in fly epithelia is the following (Figure 3):

1- Failure to sort a number of mitogenic receptors into MVBs and consequent accumulation of active signaling receptors in the endocytic pathway would provide tumor cells with self-sufficiency in growth signaling.

2- Endocytic trafficking defects would also destabilize cell-cell junctions and lead to loss of epithelial polarity, possibly triggering apoptotic response. In addition, since polarity has been shown to be important for signaling (Langevin et al., 2005; Sotillos et al., 2008), loss of polarity could also directly contribute to alter signaling in tumor cells.

3- Strong mitogenic signaling might counteract apoptotic response allowing some of the mutant cells to escape apoptotic elimination.

4- Unrestrained proliferation, freedom from the constrains of epithelia and apoptotic resistance might cooperatively provide the basis for invasive and metastatic behavior.

5- While the role of tumor-host interaction in these tumors is not clear, non-cell-autonomous promotion of apoptotic resistance and growth might play an additional role in disrupting the overall structure of the tumor-bearing epithelial organs.

While certainly incomplete, this model highlights the point that the pleiotropic defects seen when endocytic gene function is disrupted in Drosophila epithelia, which bear similarities to several hallmarks of cancer, suggest that subversion of 


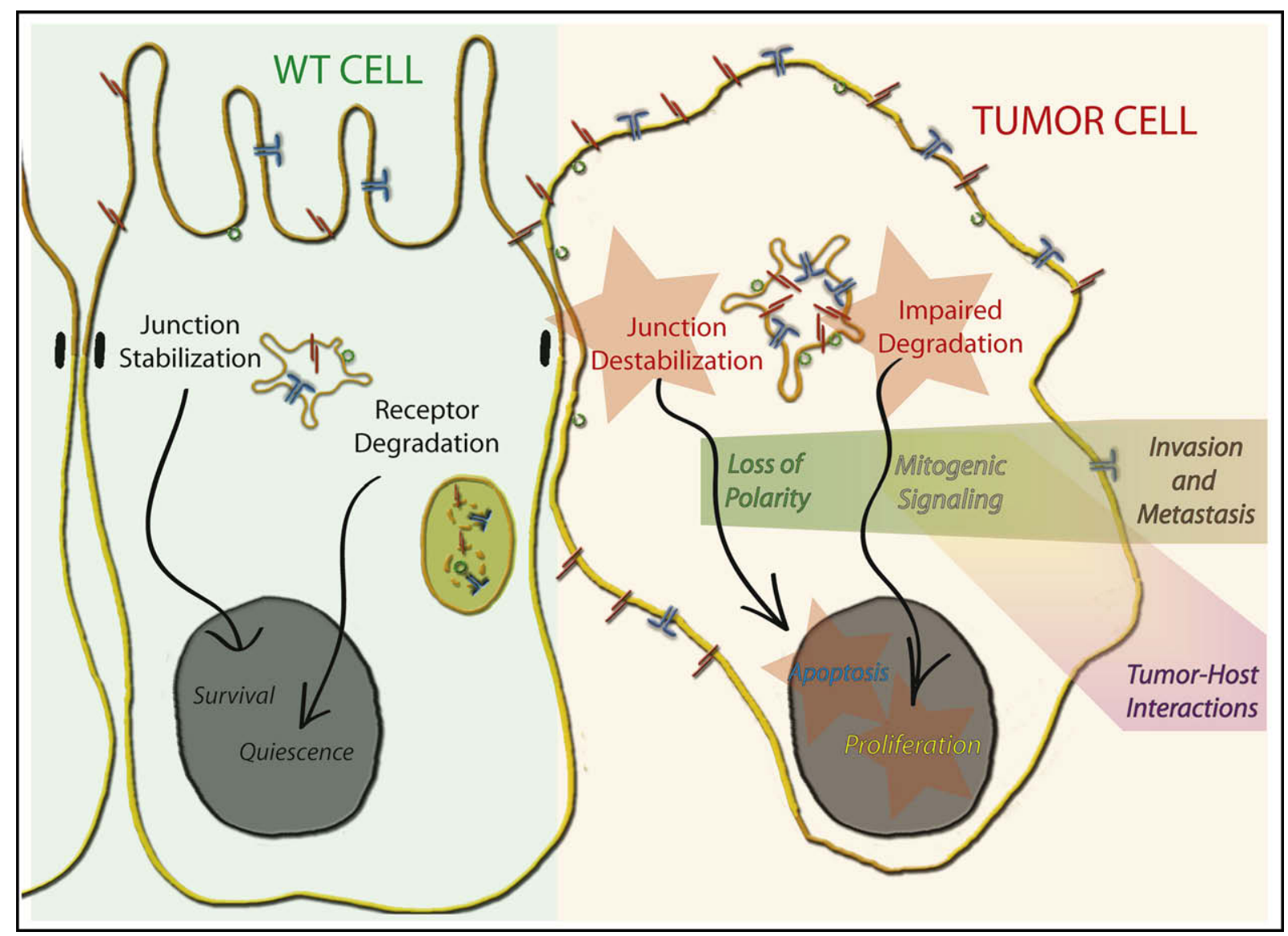

Figure 3 - Speculative model of endocytic tumor suppression circuitry. Disruption of major endocytic regulators in Drosophila initiates a tumorigenic process that displays a number of the hallmarks of human cancer. In WT epithelial cells, normal endocytic function ensures proper receptor degradation (Notch in red, EGFR in blue, membrane-associated signaling factors in green) and cell-cell junction stabilization promoting survival and quiescence (left). In tumor cells (right), increased mitogenic signaling, due to accumulation of multiple receptors in the endosomal system, contributes to acquire self-sufficiency in growth signaling. In addition, junction destabilization, due to impaired endocytic trafficking, results in loss of epithelial polarity and potential insensitivity to antigrowth signals. Loss of junctions might also relieve contact inhibition, leading to activation of apoptotic pathways. Moreover, increased mitogenic signaling and increased proliferation might help tumor cells escape apoptotic elimination. Finally, loss of polarity and increase mitogenic signaling cooperate to promote invasive and metastatic behavior in tumor cells, while non-cell-autonomous signaling between tumor cells and their surroundings represent an example of tumor-host interactions.

endocytosis might be an underappreciated but important step in cancer progression.

\section{Open questions}

The dramatic phenotypes seen upon inactivation of endocytic regulators in Drosophila underline the central importance of endocytosis in controlling both normal growth and tumor suppression, as well as potentially additional unexpected physiological processes. As this field of study is still in its infancy, a number of outstanding questions remain to be addressed in the Drosophila system. First, an important underexplored question is the extent to which expression and activity of endocytic regulators is developmentally regulated in normal tissues to control polarity, proliferation and apoptosis during organ formation. Second, it will be interesting to complete the list of endocytic regulators that act as TSGs. These studies should shed light on why some relatively early endocytic regulators, such as the MVB sorting protein Hrs (Lloyd et al., 2002; Jekely and Rorth, 2003), are not involved in tumor suppression, and why regulators of the recycling pathway, which are mutated in certain human cancer (Cheng et al., 2004; Garcia et al., 2005), have not emerged in these studies. The apparent requirement of the key recycling regulator Rab11 for cell viability may provide a partial explanation to the latter question. Finally, it will be important to identify the specific effector cargoes mistrafficked in cells mutant for each endocytic TSG, especially those that are involved in controlling polarity and apoptosis. The fact that endocytic TSG mutant cells under certain conditions escape apoptosis and overproliferate massively allows harvesting of null mutant tissue, granting the opportunity for both proteomic screening to identify mistrafficked cargoes and expression profiling to determine cellular processes altered by loss of tumor suppression.

Once the list of endocytic TSGs and cargoes involved in tumor suppression is completed, one challenge will be teasing out the relative contribution of single cargoes to the final tumor outcome. Since the phenotypic outcome of endocytic 
alterations within different tissues may depend on the tissues' intrinsic mitotic activity, polarity, and apoptotic physiology, it would be ideal to study this in multiple tissues. Such an effort will entail a systematic block of each single pathway altered in endocytic TSGs mutant cells, an analysis amenable to the power of Drosophila genetics. Finally, it will be of high interest to determine how much of the endocytic mutant tissue phenotype depends on failed degradation of signaling cargoes, as opposed to other cargo-independent functions. While little is known about these functions, it has been recently appreciated that the endosome can act as a platform to assemble and possibly deliver to the site of action molecular machines that influence diverse cellular processes including cell polarity (reviewed in Gould and Lippincott-Schwartz, 2009). Overall, future studies aimed at answering the above questions will further our knowledge of endocytic tumor suppression and broaden the list of endocytic TSGs and of the molecules and cellular processes controlled by them.

\section{Relevance of Drosophila endocytic tumorigenesis to human cancers}

What will be the relevance of studying endocytic tumor suppression in Drosophila to an understanding of human cancers? With respect to molecular similarities, to date most endocytic regulators implicated in human cancer - the ubiquitin ligase Cbl, the AP-2 interactor Numb, and the Rab5 effector Rabaptin-5, (see Mosesson et al., 2008 for an extensive list), have not been yet found in Drosophila TSG screens. However, there is some evidence for Drosophila TSGs with endocytic roles that also act as tumor suppressors in mammals. Tsg101, a component of the ESCRT-I complex, was originally identified because transplantation of tsg101-deficient cells into nude mice generated tumors (Li and Cohen, 1996). The notion that Tsg101 acts as TSG was subsequently challenged by two reports. One found that Tsg101 mutant cells in conditional knock-out animals had proliferation and survival defects but failed to develop tumors (Ruland et al., 2001; Wagner et al., 2003); the other revealed that overexpression rather than inactivation of tsg101 was observed in a subset of invasive tumors and that forced tsg101 overexpression displayed mild oncogenic effects (Oh et al., 2007). In contrast, another ESCRT-I component, vps37a/hcrp1, was recently shown to be downregulated in hepatocellular carcinoma; its inactivation relieves growth inhibition and enhances invasive ability of tumor cells (Xu et al., 2003; Bache et al., 2004). Drosophila studies suggest ways to reconcile some of these apparently contradicting data. Indeed, the fact that clones of ESCRT mutant cells in Drosophila are sensitive to apoptotic elimination but overgrow upon block of apoptosis (Thompson et al., 2005; Herz et al., 2006) predicts that the full tumorigenic potential of mammalian tsg101 cells might be hindered by apoptotic elimination. In addition, evidence in Drosophila indicates that overexpression of certain ESCRT can have a dominant negative effect (Sweeney et al., 2006; Rodahl et al., 2009). Finally, work in Drosophila might point to unexpected endocytic functions for well-studied mammalian TSGs. This is the case of NF2, a well-known human TSG with an unclear cellular function which studies in Drosophila supported its importance as a regulator of endocytosis (Maitra et al., 2006). Future studies may similarly reveal unexpected links between other known mammalian TSGs and endocytic mechanisms.

The emerging roles of endocytosis in regulating functionally conserved mitogenic signaling pathways in Drosophila underlines the relevance of the studies reviewed here to human cancers. With respect to RTK signaling, human tissue culture cells depleted of ESCRT components fail to degrade EGFR leading to increased signaling, as also seen in Drosophila (Kanazawa et al., 2003; Doyotte et al., 2005; Komada and Kitamura, 2005; Razi and Futter, 2006). Considering that human cancer cells draw on an astonishing number of ways to inactivate the machinery that ubiquitylate RTKs and sort them for degradation via MVBs (for review see Mosesson et al., 2008), Drosophila endocytic tumors might prove a valuable model for cancers that display increased RTK signaling. With respect to Notch signaling, work to ascertain the basis of ligand-independent activation in pathologic Notch signaling events in Drosophila tumors such as ESCRT mutants may inform the study of some blood cancers. Indeed, ligand-independent Notch activation is associated with $43 \%$ of T-cell acute lymphoblastic leukaemia (T-ALL) (Grabher et al., 2006; Roy et al., 2007). It will exciting to know whether the destabilizing mutations leading to ligand-independent Notch activation found in T-ALL patients affect endocytic Notch trafficking. With respect to the fact that ESCRT mutant cells induces JAK/STAT signaling non autonomously, it is interesting to note that the Drosophila Jak/STAT receptor Domeless is related to IL-6, which mediates inflammatory cytokine signaling in vertebrates (Brown et al., 2001). Inflammatory cytokines are known to be secreted by human cancer cells to modify their environment (Lin and Karin, 2007). Thus, despite differences in inflammation mechanisms in fly and vertebrates, recapitulation of simple tumor-host interactions in Drosophila allows the possibility of future genetic dissection of a heterotypic signaling event potentially relevant to tumor inflammation.

In addition to signaling, other hallmarks of human cancer observed in Drosophila endocytic tumors are the loss of epithelial polarity and escape from apoptotic elimination. With respect to polarity, downregulation of Crumbs3 correlates loss of tight junctions, impaired cell adhesion and contact inhibition, and stimulates migration and metastasis in a mouse kidney cell line model (Karp et al., 2008). Consistent with this, the Snail transcription factor, a master gene controlling EMT, represses expression of Crumbs3 (Whiteman et al., 2008). This evidence indicating that functional impairment of Crumbs might contribute to cancer progression, suggests that the study Drosophila endocytic TSGs might inform us on cancer-relevant polarity aberration. Finally, with respect to the apoptotic response, JNK signaling has been found altered in a wide number of mammalian cancers (Huang et al., 2003; Ventura et al., 2004; Hagemann et al., 2005; Kwei et al., 2006). In addition, dysregulation of the mammalian Hippo pathway has been linked to tumorigenesis (Dong et al., 2007). Thus, the tantalizing evidence that Drosophila endocytic tumor cells display opposite JNK- and Hippo-mediated apoptotic behavior, depending on their immediate environment, predicts that their future study might shed light on the mechanisms of apoptotic escape in human cancer cells.

In summary, the above evidence highlights the promise of studies of Drosophila tumorigenesis studies to illuminate 
human cancer biology. It also provides a rationale for future further screening in Drosophila and for systematic search for human homologs of Drosophila endocytic TSG whose expression is reduced in human cancers. Even in the case in which a certain endocytic TSG is found not to act as a tumor suppressor in human cancers, a comparison of the signaling profile and phenotypic pattern of Drosophila with that of existing human cancers might lead to the establishment of new predictive cancer models in Drosophila.

\section{Concluding remarks and future perspectives}

The complexity of untangling all the alterations that underlie the tumor suppressive role of endocytosis is clearly exemplified by the pleiotropy of the defects observed in Drosophila endocytic tumors. However, exploiting the advantages of Drosophila, the approaches described above provide an opportunity to systematically and comprehensively define the mitogenic, polarity and apoptotic signature of endocytic tumors. This knowledge is likely to direct the search for more human cancers in which endocytic function is subverted.

Studies on Drosophila TSGS have in the past promoted not only identification of TSGs and an understanding of their basic biology and, but have also pointed the way to possible therapeutic interventions. The seminal discovery in Drosophila that the TSGs Tsc1 and Tsc2 are mTOR signaling components led to Rapamycin treatment for Tuberous Sclerosis tumors (Kenerson et al., 2005; Lee et al., 2005; Franz et al., 2006). In much the same way, we envisage that the emerging field of endocytosis and tumor suppression in Drosophila could lead, in the years to come, towards useful strategies to develop novel anticancer drugs aimed at reactivating compromised endocytic function or inactivating potentially carcinogenic cargoes.

\section{Acknowledgments}

We apologize to our colleagues whose studies could not be covered due to space limitations. T.V. is supported by American Heart Association Award \#0825176F and by a new unit start-up grant from Associazione Italiana Ricerca contro il Cancro; D.B. is supported by National Institutes of Health grant R01GM 068675 and American Cancer Society grant RSG-07-040-01.

R E F E R E N C E S

Adachi-Yamada, T., Fujimura-Kamada, K., Nishida, Y., Matsumoto, K., 1999. Distortion of proximodistal information causes JNK-dependent apoptosis in Drosophila wing. Nature 400, 166-169.

Adachi-Yamada, T., O'Connor, M.B., 2002. Morphogenetic apoptosis: a mechanism for correcting discontinuities in morphogen gradients. Dev. Biol. 251, 74-90.

Bache, K.G., Slagsvold, T., Cabezas, A., Rosendal, K.R., Raiborg, C., Stenmark, H., 2004. The growth-regulatory protein HCRP1/hVps37A is a subunit of mammalian ESCRT-I and mediates receptor down-regulation. Mol. Biol. Cell 15, 4337-4346.
Balklava, Z., Pant, S., Fares, H., Grant, B.D., 2007. Genome-wide analysis identifies a general requirement for polarity proteins in endocytic traffic. Nat. Cell Biol. 9, 1066-1073.

Bernards, A., Hariharan, I.K., 2001. Of flies and men - studying human disease in Drosophila. Curr. Opin. Genet. Dev. 11, 274-278.

Bilder, D., Li, M., Perrimon, N., 2000. Cooperative regulation of cell polarity and growth by Drosophila tumor suppressors. Science 289, 113-116.

Bilder, D., 2004. Epithelial polarity and growth control: links from the Drosophila neoplastic tumor suppressors. Genes Dev. 18, 1909-1925.

Bissell, M.J., Radisky, D., 2001. Putting tumours in context. Nat. Rev. Cancer 1, 46-54.

Bökel, C., Schwabedissen, A., Entchev, E., Renaud, O., GonzálezGaitán, M., 2006. Sara endosomes and the maintenance of Dpp signaling levels across mitosis. Science 314, 1135-1139.

Brown, S., Hu, N., Hombría, J.C., 2001. Identification of the first invertebrate interleukin JAK/STAT receptor, the Drosophila gene domeless. Curr. Biol. 11, 1700-1705.

Brumby, A.M., Richardson, H.E., 2003. Scribble mutants cooperate with oncogenic Ras or Notch to cause neoplastic overgrowth in Drosophila. EMBO J. 22, 5769-5779.

Brumby, A.M., Richardson, H.E., 2005. Using Drosophila melanogaster to map human cancer pathways. Nat. Rev. Cancer 5, 626-639.

Bryant, P.J., Schubiger, G., 1971. Giant and duplicated imaginal discs in a new lethal mutant of Drosophila melanogaster. Dev. Biol. 24, 233-263.

Chao, J.L., Tsai, Y.C., Chiu, S.J., Sun, Y.H., 2004. Localized notch signal acts through eyg and upd to promote global growth in Drosophila eye. Development 131, 3839-3847.

Cheng, K.W., Lahad, J.P., Kuo, W.L., Lapuk, A., Yamada, K., Auersperg, N., Liu, J., Smith-McCune, K., Lu, K.H., Fishman, D., et al., 2004. The RAB25 small GTPase determines aggressiveness of ovarian and breast cancers. Nat. Med. 10, 1251-1256.

Childress, J.L., Acar, M., Tao, C., Halder, G., 2006. Lethal giant discs, a novel C2-domain protein, restricts notch activation during endocytosis. Curr. Biol. 16, 2228-2233.

Coumailleau, F., Fürthauer, M., Knoblich, J.A., GonzálezGaitán, M., 2009. Directional delta and notch trafficking in Sara endosomes during asymmetric cell division. Nature.

Curto, M., Cole, B.K., Lallemand, D., Liu, C.H., McClatchey, A.I., 2007. Contact-dependent inhibition of EGFR signaling by Nf2/ Merlin. J. Cell Biol. 177, 893-903.

Devergne, O., Ghiglione, C., Noselli, S., 2007. The endocytic control of JAK/STAT signalling in Drosophila. J. Cell Sci. 120, 3457-3464.

Doherty, G.J., McMahon, H.T., 2009. Mechanisms of endocytosis. Annu. Rev. Biochem.

Dong, J., Feldmann, G., Huang, J., Wu, S., Zhang, N., Comerford, S.A., Gayyed, M.F., Anders, R.A., Maitra, A., Pan, D., 2007. Elucidation of a universal size-control mechanism in Drosophila and mammals. Cell 130, 1120-1133.

Doyotte, A., Russell, M.R., Hopkins, C.R., Woodman, P.G., 2005. Depletion of TSG101 forms a mammalian "Class E" compartment: a multicisternal early endosome with multiple sorting defects. J. Cell Sci. 118, 3003-3017.

Dubois, L., Lecourtois, M., Alexandre, C., Hirst, E., Vincent, J.P., 2001. Regulated endocytic routing modulates wingless signaling in Drosophila embryos. Cell 105, 613-624.

Edgar, B.A., 2006. From cell structure to transcription: Hippo forges a new path. Cell 124, 267-273.

Fortini, M.E., Skupski, M.P., Boguski, M.S., Hariharan, I.K., 2000. A survey of human disease gene counterparts in the Drosophila genome. J. Cell Biol. 150, F23-F30.

Fortini, M.E., Bilder, D. Endocytic regulation of notch signaling. Curr Opin Genet Dev., in press (2009 May 15 e-pub ahead of print).

Franz, D.N., Leonard, J., Tudor, C., Chuck, G., Care, M., Sethuraman, G., Dinopoulos, A., Thomas, G., Crone, K.R., 2006. 
Rapamycin causes regression of astrocytomas in tuberous sclerosis complex. Ann. Neurol. 59, 490-498.

Fuwa, T.J., Hori, K., Sasamura, T., Higgs, J., Baron, M., Matsuno, K., 2006. The first deltex null mutant indicates tissue-specific deltex-dependent notch signaling in Drosophila. Mol. Genet. Genomics 275, 251-263.

Gallagher, C.M., Knoblich, J.A., 2006. The conserved c2 domain protein lethal (2) giant discs regulates protein trafficking in Drosophila. Dev. Cell 11, 641-653.

Garcia, M.J., Pole, J.C., Chin, S.F., Teschendorff, A., Naderi, A., Ozdag, H., Vias, M., Kranjac, T., Subkhankulova, T., Paish, C., et al., 2005. A $1 \mathrm{Mb}$ minimal amplicon at 8p11-12 in breast cancer identifies new candidate oncogenes. Oncogene 24, 5235-5245.

Gateff, E., Schneiderman, H., 1967. Developmental studies of a new mutant of Drosophila melanogaster: lethal malignant brain tumor (1(2)gl 4). Am. Zool. 7, 760.

Gateff, E., 1978. Malignant neoplasms of genetic origin in Drosophila melanogaster. Science 200, 1448-1459.

Georgiou, M., Marinari, E., Burden, J., Baum, B., 2008. Cdc42, Par6, and aPKC regulate Arp2/3-mediated endocytosis to control local adherens junction stability. Curr. Biol. 18, 1631-1638.

Gould, G., Lippincott-Schwartz, J., 2009. New roles for endosomes: from vesicular carriers to multi-purpose platforms. Nat. Rev. Mol. Cell Biol. 10, 287-292.

Grabher, C., von Boehmer, H., Look, A.T., 2006. Notch 1 activation in the molecular pathogenesis of T-cell acute lymphoblastic leukaemia. Nat. Rev. Cancer 6, 347-359.

Hagemann, T., Wilson, J., Kulbe, H., Li, N.F., Leinster, D.A., Charles, K., Klemm, F., Pukrop, T., Binder, C., Balkwill, F.R., 2005. Macrophages induce invasiveness of epithelial cancer cells via NF-kappa B and JNK. J. Immunol. 175, 1197-1205.

Hamaratoglu, F., Willecke, M., Kango-Singh, M., Nolo, R., Hyun, E., Tao, C., Jafar-Nejad, H., Halder, G., 2006. The tumoursuppressor genes NF2/Merlin and Expanded act through Hippo signalling to regulate cell proliferation and apoptosis. Nat. Cell Biol. 8, 27-36.

Hanahan, D., Weinberg, R.A., 2000. The hallmarks of cancer. Cell 100, 57-70.

Hariharan, I.K., Bilder, D., 2006. Regulation of imaginal disc growth by tumor-suppressor genes in Drosophila. Annu. Rev. Genet. 40, 335-361.

Harris, K.P., Tepass, U., 2008. Cdc42 and Par proteins stabilize dynamic adherens junctions in the Drosophila neuroectoderm through regulation of apical endocytosis. J. Cell Biol. 183, 1129-1143.

Herz, H.M., Chen, Z., Scherr, H., Lackey, M., Bolduc, C., Bergmann, A., 2006. vps25 mosaics display non-autonomous cell survival and overgrowth, and autonomous apoptosis. Development 133, 1871-1880.

Hori, K., Fostier, M., Ito, M., Fuwa, T.J., Go, M.J., Okano, H., Baron, M., Matsuno, K., 2004. Drosophila deltex mediates suppressor of hairless-independent and late-endosomal activation of notch signaling. Development 131, 5527-5537.

Huang, S., Shu, L., Dilling, M.B., Easton, J., Harwood, F.C., Ichijo, H., Houghton, P.J., 2003. Sustained activation of the JNK cascade and rapamycin-induced apoptosis are suppressed by p53/p21(Cip1). Mol. Cell 11, 1491-1501.

Humbert, P.O., Russell, S.M., Richardson, H.E., 2003. Dlg, Scribble and Lgl in cell polarity, cell proliferation and cancer. Bioessays 25, 542-553.

Igaki, T., Pagliarini, R.A., Xu, T., 2006. Loss of cell polarity drives tumor growth and invasion through JNK activation in Drosophila. Curr. Biol. 16, 1139-1146.

Igaki, T., Pastor-Pareja, J.C., Aonuma, H., Miura, M., Xu, T., 2009. Intrinsic tumor suppression and epithelial maintenance by endocytic activation of Eiger/TNF signaling in Drosophila. Dev. Cell 16, 458-465.
Jaekel, R., Klein, T., 2006. The Drosophila notch inhibitor and tumor suppressor gene lethal (2) giant discs encodes a conserved regulator of endosomal trafficking. Dev. Cell 11, 655-669.

Jekely, G., Rorth, P., 2003. Hrs mediates downregulation of multiple signalling receptors in Drosophila. EMBO Rep. 4, 1163-1168.

Jékely, G., Sung, H.H., Luque, C.M., Rørth, P., 2005. Regulators of endocytosis maintain localized receptor tyrosine kinase signaling in guided migration. Dev. Cell 9, 197-207.

Johnson, K.C., Kissil, J.L., Fry, J.L., Jacks, T., 2002. Cellular transformation by a FERM domain mutant of the Nf2 tumor suppressor gene. Oncogene 21, 5990-5997.

Kanazawa, C., Morita, E., Yamada, M., Ishii, N., Miura, S., Asao, H., Yoshimori, T., Sugamura, K., 2003. Effects of deficiencies of STAMs and Hrs, mammalian class E Vps proteins, on receptor downregulation. Biochem. Biophys. Res. Commun. 309, 848-856.

Karp, C.M., Tan, T.T., Mathew, R., Nelson, D., Mukherjee, C., Degenhardt, K., Karantza-Wadsworth, V., White, E., 2008. Role of the polarity determinant crumbs in suppressing mammalian epithelial tumor progression. Cancer Res. 68, 4105-4115.

Kenerson, H., Dundon, T.A., Yeung, R.S., 2005. Effects of rapamycin in the Eker rat model of tuberous sclerosis complex. Pediatr. Res. 57, 67-75.

Komada, M., Kitamura, N., 2005. The Hrs/STAM complex in the downregulation of receptor tyrosine kinases. J. Biochem. 137, 1-8.

Kopan, R., Ilagan, M.X., 2009. The canonical notch signaling pathway: unfolding the activation mechanism. Cell 137, 216-233.

Kwei, K.A., Finch, J.S., Ranger-Moore, J., Bowden, G.T., 2006. The role of Rac1 in maintaining malignant phenotype of mouse skin tumor cells. Cancer Lett. 231, 326-338.

Langevin, J., Le Borgne, R., Rosenfeld, F., Gho, M., Schweisguth, F., Bellaïche, Y., 2005. Lethal giant larvae controls the localization of notch-signaling regulators numb, neuralized, and Sanpodo in Drosophila sensory-organ precursor cells. Curr. Biol. 15, 955-962.

Lee, L., Sudentas, P., Donohue, B., Asrican, K., Worku, A., Walker, V., Sun, Y., Schmidt, K., Albert, M.S., El-Hashemite, N., et al., 2005. Efficacy of a rapamycin analog (CCI-779) and IFNgamma in tuberous sclerosis mouse models. Genes Chromosomes Cancer 42, 213-227.

Leibfried, A., Fricke, R., Morgan, M.J., Bogdan, S., Bellaiche, Y., 2008. Drosophila Cip4 and WASp define a branch of the Cdc42Par6-aPKC pathway regulating E-cadherin endocytosis. Curr. Biol. 18, 1639-1648.

Li, L., Cohen, S.N., 1996. Tsg101: a novel tumor susceptibility gene isolated by controlled homozygous functional knockout of allelic loci in mammalian cells. Cell 85, 319-329.

Lin, W.W., Karin, M., 2007. A cytokine-mediated link between innate immunity, inflammation, and cancer. J. Clin. Invest. 117, 1175-1183.

Lloyd, T.E., Atkinson, R., Wu, M., Zhou, Y., Pennetta, G., Bellen, H.J., 2002. Hrs regulates endosome membrane invagination and tyrosine kinase receptor signaling in Drosophila. Cell 108, 261-269.

Lu, H., Bilder, D., 2005. Endocytic control of epithelial polarity and proliferation in Drosophila. Nat. Cell Biol. 7, 1132-1139.

Maitra, S., Kulikauskas, R.M., Gavilan, H., Fehon, R.G., 2006. The tumor suppressors Merlin and Expanded function cooperatively to modulate receptor endocytosis and signaling. Curr. Biol. 16, 702-709.

Marois, E., Mahmoud, A., Eaton, S., 2006. The endocytic pathway and formation of the wingless morphogen gradient. Development 133, 307-317.

McClatchey, A.I., Fehon, R.G., 2009. Merlin and the ERM proteins regulators of receptor distribution and signaling at the cell cortex. Trends Cell Biol.

Menut, L., Vaccari, T., Dionne, H., Hill, J., Wu, G., Bilder, D., 2007. A mosaic genetic screen for Drosophila neoplastic tumor 
suppressor genes based on defective pupation. Genetics 177, 1667-1677.

Miaczynska, M., Pelkmans, L., Zerial, M., 2004. Not just a sink: endosomes in control of signal transduction. Curr. Opin. Cell Biol. 16, 400-406.

Moberg, K.H., Schelble, S., Burdick, S.K., Hariharan, I.K., 2005. Mutations in erupted, the Drosophila ortholog of mammalian tumor susceptibility gene 101, elicit non-cell-autonomous overgrowth. Dev. Cell 9, 699-710.

Morrison, H.A., Dionne, H., Rusten, T.E., Brech, A., Fisher, W.W., Pfeiffer, B.D., Celniker, S.E., Stenmark, H., Bilder, D., 2008. Regulation of early endosomal entry by the Drosophila tumor suppressors Rabenosyn and Vps45. Mol. Biol. Cell.

Mosesson, Y., Mills, G., Yarden, Y., 2008. Derailed endocytosis: an emerging feature of cancer. Nat. Rev. Cancer 8, 835-850.

Mukherjee, A., Veraksa, A., Bauer, A., Rosse, C., Camonis, J., Artavanis-Tsakonas, S., 2005. Regulation of notch signalling by non-visual beta-arrestin. Nat. Cell Biol. 7, 1191-1201.

Oh, K.B., Stanton, M.J., West, W.W., Todd, G.L., Wagner, K.U., 2007. Tsg101 is upregulated in a subset of invasive human breast cancers and its targeted overexpression in transgenic mice reveals weak oncogenic properties for mammary cancer initiation. Oncogene 26, 5950-5959.

Pagliarini, R.A., Xu, T., 2003. A genetic screen in Drosophila for metastatic behavior. Science 302, 1227-1231.

Pai, L.M., Barcelo, G., Schüpbach, T., 2000. D-cbl, a negative regulator of the Egfr pathway, is required for dorsoventral patterning in Drosophila oogenesis. Cell 103, 51-61.

Pasternak, S.H., Bagshaw, R.D., Guiral, M., Zhang, S., Ackerley, C.A., Pak, B.J., Callahan, J.W., Mahuran, D.J., 2003. Presenilin-1, nicastrin, amyloid precursor protein, and gamma-secretase activity are co-localized in the lysosomal membrane. J. Biol. Chem. 278, 26687-26694.

Piddini, E., Marshall, F., Dubois, L., Hirst, E., Vincent, J.P., 2005. Arrow (LRP6) and Frizzled2 cooperate to degrade wingless in Drosophila imaginal discs. Development 132, 5479-5489.

Razi, M., Futter, C.E., 2006. Distinct roles for Tsg101 and Hrs in multivesicular body formation and inward vesiculation. Mol. Biol. Cell 17, 3469-3483.

Rives, A.F., Rochlin, K.M., Wehrli, M., Schwartz, S.L., DiNardo, S., 2006. Endocytic trafficking of wingless and its receptors, Arrow and DFrizzled-2, in the Drosophila wing. Dev. Biol. 293, 268-283.

Rodahl, L.M., Haglund, K., Sem-Jacobsen, C., Wendler, F., Vincent, J.P., Lindmo, K., Rusten, T.E., Stenmark, H., 2009. Disruption of Vps4 and JNK function in Drosophila causes tumour growth. PLoS ONE 4, e4354.

Roy, M., Pear, W.S., Aster, J.C., 2007. The multifaceted role of notch in cancer. Curr. Opin. Genet. Dev. 17, 52-59.

Ruland, J., Sirard, C., Elia, A., MacPherson, D., Wakeham, A., Li, L., de la Pompa, J.L., Cohen, S.N., Mak, T.W., 2001. p. 53 accumulation, defective cell proliferation, and early embryonic lethality in mice lacking tsg101. Proc. Natl. Acad. Sci. USA 98, 1859-1864.

Rusten, T.E., Rodahl, L.M., Pattni, K., Englund, C., Samakovlis, C., Dove, S., Brech, A., Stenmark, H., 2006. Fab1 phosphatidylinositol 3-phosphate 5-kinase controls trafficking but not silencing of endocytosed receptors. Mol. Biol. Cell 17, 3989-4001.

Sakata, T., Sakaguchi, H., Tsuda, L., Higashitani, A., Aigaki, T., Matsuno, K., Hayashi, S., 2004. Drosophila Nedd4 regulates endocytosis of notch and suppresses its ligand-independent activation. Curr. Biol. 14, 2228-2236.

Saksena, S., Sun, J., Chu, T., Emr, S.D., 2007. ESCRTing proteins in the endocytic pathway. Trends Biochem. Sci. 32, 561-573.

Seto, E.S., Bellen, H.J., 2006. Internalization is required for proper wingless signaling in Drosophila melanogaster. J. Cell Biol. 173, 95-106.
Shestopal, S.A., Makunin, I.V., Belyaeva, E.S., Ashburner, M., Zhimulev, I.F., 1997. Molecular characterization of the deep orange (dor) gene of Drosophila melanogaster. Mol. Gen. Genet. 253, 642-648.

Sotillos, S., Díaz-Meco, M.T., Moscat, J., Castelli-Gair Hombría, J., 2008. Polarized subcellular localization of Jak/STAT components is required for efficient signaling. Curr. Biol. 18, 624-629.

Spang, A., 2009. On the fate of early endosomes. Biol. Chem.

St Johnston, M.A., 2002. The art and design of genetic screens: Drosophila melanogaster. Nat. Rev. Genet. 3, 176-188.

Sweeney, N., Brenman, J., Jan, Y., Gao, F., 2006. The coiled-coil protein shrub controls neuronal morphogenesis in Drosophila. Curr. Biol. 16, 1006-1011.

Thompson, B.J., Mathieu, J., Sung, H.H., Loeser, E., Rorth, P., Cohen, S.N., 2005. Tumor suppressor properties of the ESCRT-II complex component Vps25 in Drosophila. Dev. Cell 9, 711-720.

Tien, A.C., Rajan, A., Bellen, H.J., 2009. A notch updated. J. Cell Biol. 184, 621-629.

Tsai, Y.C., Sun, Y.H., 2004. Long-range effect of upd, a ligand for Jak/STAT pathway, on cell cycle in Drosophila eye development. Genesis 39, 141-153.

Uhlirova, M., Bohmann, D., 2006. JNK- and Fos-regulated Mmp1 expression cooperates with Ras to induce invasive tumors in Drosophila. EMBO J. 25, 5294-5304.

Vaccari, T., Bilder, D., 2005. The Drosophila tumor suppressor vps25 prevents nonautonomous overproliferation by regulating notch trafficking. Dev. Cell 9, 687-698.

Vaccari, T., Lu, H., Kanwar, R., Fortini, M.E., Bilder, D., 2008. Endosomal entry regulates notch receptor activation in Drosophila melanogaster. J. Cell Biol. 180, 755-762.

Vaccari, T., Rusten, T.E., Menut, L., Nezis, I., Brech, A., Stenmark, H., Bilder, D. Comparative analysis of ESCRT -I, -II, -III function in Drosophila by efficient isolation of ESCRT mutants. J. Cell Sci., in press.

Ventura, J.J., Cogswell, P., Flavell, R.A., Baldwin, A.S., Davis, R.J., 2004. JNK potentiates TNF-stimulated necrosis by increasing the production of cytotoxic reactive oxygen species. Genes Dev. 18, 2905-2915.

Vidal, M., Cagan, R.L., 2006. Drosophila models for cancer research. Curr. Opin. Genet. Dev. 16, 10-16.

Wagner, K.U., Krempler, A., Qi, Y., Park, K., Henry, M.D., Triplett, A.A., Riedlinger, G., Rucker, I.E., Hennighausen, L., 2003. Tsg101 is essential for cell growth, proliferation, and cell survival of embryonic and adult tissues. Mol. Cell. Biol. 23, 150-162.

Warner, T.S., Sinclair, D.A., Fitzpatrick, K.A., Singh, M., Devlin, R.H., Honda, B.M., 1998. The light gene of Drosophila melanogaster encodes a homologue of VPS41, a yeast gene involved in cellular-protein trafficking. Genome 41, 236-243.

Whiteman, E.L., Liu, C.J., Fearon, E.R., Margolis, B., 2008. The transcription factor snail represses Crumbs 3 expression and disrupts apico-basal polarity complexes. Oncogene 27, 3875-3879.

Wilkin, M., Tongngok, P., Gensch, N., Clemence, S., Motoki, M., Yamada, K., Hori, K., Taniguchi-Kanai, M., Franklin, E., Matsuno, K., et al., 2008. Drosophila HOPS and AP-3 complex genes are required for a deltex-regulated activation of notch in the endosomal trafficking pathway. Dev. Cell 15, 762-772.

Wilkin, M.B., Carbery, A.M., Fostier, M., Aslam, H., Mazaleyrat, S.L., Higgs, J., Myat, A., Evans, D.A., Cornell, M., Baron, M., 2004. Regulation of notch endosomal sorting and signaling by Drosophila Nedd4 family proteins. Curr. Biol. 14, 2237-2244.

Willecke, M., Hamaratoglu, F., Kango-Singh, M., Udan, R., Chen, C.L., Tao, C., Zhang, X., Halder, G., 2006. The fat cadherin acts through the hippo tumor-suppressor pathway to regulate tissue size. Curr. Biol. 16, 2090-2100.

Xu, Z., Liang, L., Wang, H., Li, T., Zhao, M., 2003. HCRP1, a novel gene that is downregulated in hepatocellular carcinoma, encodes a growth-inhibitory protein. Biochem. Biophys. Res. Commun. 311, 1057-1066. 